\title{
"Les souris sont de bons modèles»
}

\section{Daniel Lüthi}

Journaliste indépendant et photographe, conseiller médiatique, Berne

1 Gollwitzer ES et al.

Lung microbiota promotes tolerance to allergens in neonates via PD-L1.

Nat Med. 2014:20(6):642-7.
C'est l'histoire des souris et des hommes. Et d'une jeune femme qui a déjà fait pas mal de choses dans sa vie. On vient juste de lui décerner le prix Pfizer de la recherche pour ses travaux. "Une belle cerise sur le gâteau», déclare E. Gollwitzer. «Mais la vraie gratification est de pouvoir publier les conclusions de mes travaux sous mon propre nom» [1].

\section{Fleurs, arbres... et bactéries}

E. Gollwitzer est biologiste mais travaille en fait pour la médecine. "Je ne corresponds pas du tout au cliché de la biologiste parcourant champs et forêts en Birken- stock», affirme-t-elle. «Dès le départ, il était clair que je ne voulais pas étudier les insectes.» Certes, pendant sa formation elle a examiné les fleurs et les arbres, mais pour son bachelor et son master elle était déjà passée à la virologie. «Il s'agissait des résistances du VIH aux médicaments. Je voulais découvrir comment notre système immunitaire, nos défenses corporelles, luttaient contre les intrus. On parlait déjà là de mécanismes."

Elle est toujours sur le même sujet. Mais elle s'occupe surtout de bactéries désormais. Et de souris. Concrètement: "Avec une pipette, nous injectons pendant quelques semaines des allergènes dans le nez de nos

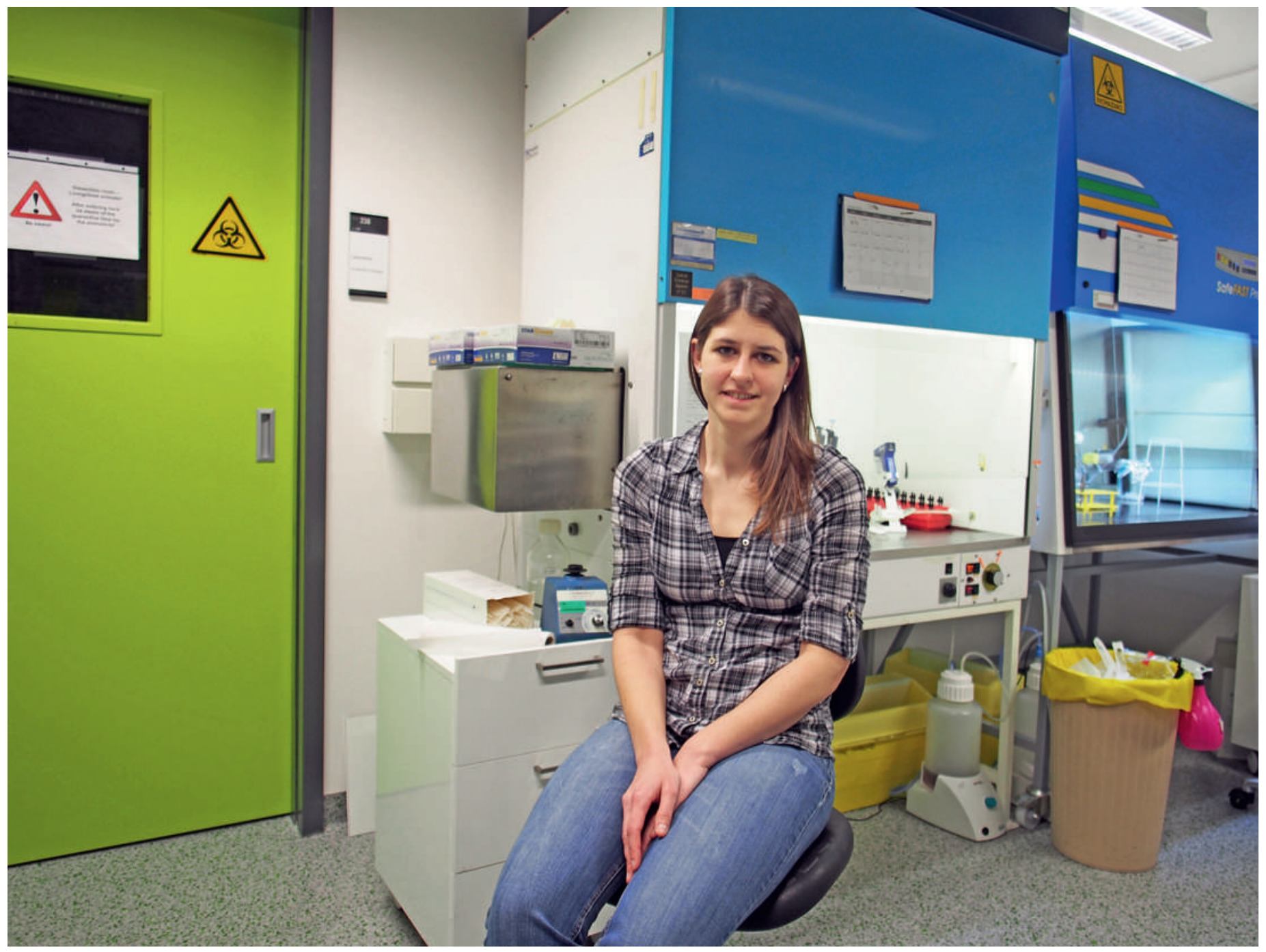


souris, un peu des acariens de la poussière, présents partout dans notre environnement, par exemple.» Comme les hommes, les souris développent alors des allergies. «Les plus jeunes réagissent plus violemment. Nous voulons savoir pourquoi et découvrir ce qui se passe dans les poumons. Les cellules $\mathrm{T}$ régulatrices jouent un rôle décisif. Elles ne sont induites qu'après les deux premières semaines d'existence des souris, et par les bactéries qui peuplent notre organisme. Elles sont là pour réduire notre réponse immunitaire.» E. Gollwitzer a transféré ces cellules $\mathrm{T}$ de souris adultes sur des souris nouvellement nées: la réaction allergique aux acariens de la poussière était contenue. En d'autres termes, les premiers mois suivant la naissance déterminent si les individus vont développer de l'asthme.

\section{Industrie pharmaceutique et indépendance}

Quel pourrait être l'intérêt de ce résultat? Le groupe pharmaceutique Pfizer écrit: «Dans l'avenir, nous pourrions stimuler naturellement l'organisme du bébé, avec un 'cocktail de bactéries', pour qu'il produise ces cellules T régulatrices. Cela pourrait prévenir l'arrivée de l'asthme, un peu comme un vaccin.»

Le groupe pharmaceutique, qui décerne maintenant des prix et sponsorise des travaux, pourrait un jour gagner de l'argent avec les résultats de ces recherches. Des chercheurs comme E. Gollwitzer pourraient-ils y perdre leur indépendance? «Mon travail n'implique aucun conflit», déclare-t-elle, «et je veux dans tous les cas conserver une certaine liberté. Je veux pouvoir décider seule de la direction de mes recherches.» Cela fait partie de son ambition, à laquelle elle ne fait aucune concession: "Je veux faire bien les choses, publier mes résultats, et générer entre autres de nouveaux fonds pour notre recherche.»

Les 15000 francs du prix, gagnés à titre personnel, sont d'abord déposés sur un compte privé. Leur usage n'est pas encore clair. E. Gollwitzer n'a quasiment pas d'exigences ni de rêves matériels. «Cet argent servira peutêtre à rester un peu plus longtemps à Lausanne, car je me plais ici.»

\section{Des souris et des hommes}

La cause première en est le travail, qui détermine largement la vie de la jeune femme. Elle raconte qu'il lui est déjà arrivé de travailler sept jours sur sept, avant une publication par exemple. «Ou parce que je dois surveiller nos souris.»

L'expression "nos souris» sonne presque tendrement, comme s'il s'agissait d'animaux domestiques. Naturel-

\section{Eva Gollwitzer}

Dr Eva Gollwitzer, PhD, est née en 1986 à Fürth (Bavière, Allemagne), où elle a suivi sa scolarité. Elle a passé bachelor et master dans la proche université d'Erlangen-Nuremberg. Puis elle est venue comme doctorante à Lausanne, où elle travaille depuis 2010 pour I'université et le CHUV. Elle est post-doctorante depuis I'an dernier. Elle a obtenu cette année le prix Pfizer de la recherche, avec d'autres jeunes chercheuses et chercheurs. Elle vit avec son partenaire à Lausanne.

lement, c'est une autre relation, déclare la biologiste, mais tout de même: «Nous passons beaucoup de temps avec nos animaux et devons surveiller tous les jours s'ils vont bien. Car les souris, ce n'est pas du matériel, mais des êtres vivants. Malheureusement, nous ne pouvons pas faire de recherche sans elles. Elles nous aident à découvrir certains mécanismes. Ce sont de bons modèles.»

Notamment quand il s'agit de créer les fondements de nouveaux médicaments, de lutter contre des maladies ou de les prévenir. Les souris sont donc au service des hommes. «Je ne mettrais pas nos souris à disposition pour développer un nouveau rouge à lèvres ou une «Dès le départ, il était clair que je ne voulais pas étudier des insectes."

crème antirides», relativise E. Gollwitzer. «Mais les allergies se développent, et pour les combattre, nous devons comprendre pourquoi. C'est clair, les bactéries nous sont nécessaires.» Rien de nouveau. La jeune femme complète: «Nous savons depuis longtemps qu'un enfant a besoin de jouer dans la poussière. Mais jusque-là, nous ne savions pas exactement comment ce lien fonctionnait.»

Il reste beaucoup à découvrir dans le secteur des allergies et de l'asthme, et elle travaille sur une niche. "Ce sujet est intéressant et concerne de nombreuses personnes, pourtant nous ne pouvons toujours rien faire concrètement.» On sait une chose: "A la naissance, nous sommes stériles. Puis nous sommes colonisés par les bactéries. Notre microbiome se constitue dans nos trois premières années. Nous avons constaté que nous avions besoin de ces bactéries, ces cellules $\mathrm{T}$ régulatrices, pour notre santé.» Un environnement trop propre et stérile n'est pas bon. "Mais il n'est pas non plus nécessaire de prescrire des antibiotiques trop tôt.» 


\section{Recherche et médecine}

Pourquoi E. Gollwitzer n'est-elle pas devenue médecin? "Je ne voulais pas assumer la responsabilité du contact direct avec le patient», répond-elle, "je préfère travailler sur les bases et agir indirectement sur la médecine.» Une action parfois distanciée, mais jamais asociale. Le travail en équipe est très important pour elle. "Dans certains laboratoires, les personnes sont instrumentalisées les unes contre les autres pour accélérer les choses. Cela peut aboutir à une ambition narcissique. Dans ce laboratoire, nous préférons travailler ensemble.»

\section{«Les souris, ce n'est pas du matériel.»}

Pour elle, la recherche est en effet une sorte de course, mais c'est en travaillant en équipe qu'on est le plus rapide. La jeune chercheuse fait par exemple régulièrement appel à des médecins, pneumologues et néonatologistes expérimentés. «Une bonne interaction est essentielle», explique-t-elle. "Les chercheurs ont besoin des médecins et vice versa.»

Elle apprécie aussi que son quotidien soit varié: le passage de la pratique à la théorie, de la pipette au microscope, de l'ordinateur mort aux souris vivantes. De l'université à l'hôpital et de la biologie à la médecine.

\section{Travail et famille}

A-t-elle encore de la place pour envisager une famille? "Absolument», répond-elle sans hésiter. Son travail lui laisse une grande souplesse, et elle peut aussi être sur l'ordinateur à la maison. «Tout est une question d'organisation. Et dans un couple, ce ne doit pas toujours être le même qui fait des concessions.» En tant que médecin, d'après elle, il serait beaucoup plus difficile de vivre comme ça.

De quoi cette jeune chercheuse a-t-elle besoin dans sa vie, en dehors de son travail et des succès qui y sont liés? «Une journée dans les montagnes de temps en temps», répond-elle simplement, des moments avec son partenaire, ingénieur informaticien, et avec ses amis.

En milieu d'année, E. Gollwitzer aura 30 ans. Seulement 30 ans, a-t-on tendance à penser quand on voit son parcours, considérant le fait que cette jeune femme encadre et instruit déjà les doctorants qui arrivent.

Que souhaite-t-elle pour ses 30 ans? Elle réfléchit rapidement et répond: "Que cela continue aussi bien que cela a commencé.»

\section{La prochaine "Rencontre avec...»}

A la fin de chaque mois, le Bulletin des médecins suisses présente une personnalité qui s'implique dans la santé publique. En mai, Daniel Lüthi parlera de sa rencontre avec Dr Jörg Peltzer, médecin-chef en chirurgie à Delémont et patron de la chirurgie des accidents en Ethiopie. 